\title{
Candida auris Outbreak in a COVID-19 Specialty Care Unit - Florida, July-August 2020
}

\begin{abstract}
Christopher Prestel, $\mathrm{MD}^{1,2}$; Erica Anderson, $\mathrm{MPH}^{2}$; Kaitlin Forsberg, $\mathrm{MPH}^{3}$; Meghan Lyman, $\mathrm{MD}^{3}$; Marie A. de Perio, $\mathrm{MD}^{4,5}$; David Kuhar, $\mathrm{MD}^{1}$;
\end{abstract} Kendra Edwards ${ }^{6}$; Maria Rivera, $\mathrm{MPH}^{2}$; Alicia Shugart, $\mathrm{MA}^{1}$; Maroya Walters, $\mathrm{PhD}^{1}$; Nychie Q. Dotson, $\mathrm{PhD}^{2}$

\begin{abstract}
On January 8, 2021, this report was posted as an MMWR Early Release on the MMWR website (https://www.cdc.gov/mmwr).
\end{abstract}

In July 2020, the Florida Department of Health was alerted to three Candida auris bloodstream infections and one urinary tract infection in four patients with coronavirus disease 2019 (COVID-19) who received care in the same dedicated COVID-19 unit of an acute care hospital (hospital A). C. auris is a multidrug-resistant yeast that can cause invasive infection. Its ability to colonize patients asymptomatically and persist on surfaces has contributed to previous $C$. auris outbreaks in health care settings (1-7). Since the first $C$. auris case was identified in Florida in 2017, aggressive measures have been implemented to limit spread, including contact tracing and screening upon detection of a new case. Before the COVID-19 pandemic, hospital A conducted admission screening for $C$. auris and admitted colonized patients to a separate dedicated ward.

Hospital A's COVID-19 unit spanned five wings on four floors, with 12-20 private, intensive care-capable rooms per wing. Only patients with positive test results for SARS-CoV-2, the virus that causes COVID-19, at the time of admission were admitted to this unit. After patient discharge, room turnover procedures included thorough cleaning of all surfaces and floor and ultraviolet disinfection. In response to the four clinical $C$. auris infections, unit-wide point prevalence surveys to identify additional hospitalized patients colonized with $C$. auris were conducted during August 4-18; patients on all four floors were screened sequentially and rescreened only if their initial result was indeterminate. Hospital A's infection prevention team, the Florida Department of Health, and CDC performed a joint investigation focused on infection prevention and control at hospital A that included observation of health care personnel (HCP) use of personal protective equipment (PPE), contact with and disinfection of shared medical equipment, hand hygiene, and supply storage. This activity was reviewed by CDC and was conducted consistent with applicable federal law and CDC policy.*

Among 67 patients admitted to the COVID-19 unit and screened during point prevalence surveys, 35 (52\%) received positive test results. Mean age of colonized patients was 69 years (range $=38-101$ years) and 60\% were male. Six (17\%) colonized patients later had clinical cultures that grew $C$. auris.

\footnotetext{
* 45 C.F.R. part 46.102(1)(2), 21 C.F.R. part 56; 42 U.S.C. Sect. 241(d); 5 U.S.C. Sect. 552a; 44 U.S.C. Sect. 3501 et seq.
}

Among patients screened who had available medical records (20), two (10\%) were admitted directly from a long-term care facility and eight (40\%) died within 30 days of screening, but whether $C$. auris contributed to death is unknown (Table).

$\mathrm{HCP}$ in the COVID-19 unit were observed wearing multiple layers of gowns and gloves during care of COVID-19 patients. HCP donned eye protection, an N95 respirator, a cloth isolation gown, gloves, a bouffant cap, and shoe covers on entry to the COVID-19 unit; these were worn during the entire shift. A second, disposable isolation gown and pair of gloves were donned before entering individual patient rooms, then doffed and discarded upon exit. Alcohol-based hand sanitizer was used on gloved hands after doffing outer gloves. HCP removed all PPE and performed hand hygiene before exiting the unit.

Investigators observed multiple opportunities for contamination of the base layer of gown and gloves during doffing and through direct contact with the patient care environment or potentially contaminated surfaces such as mobile computers. Mobile computers and medical equipment were not always disinfected between uses, medical supplies (e.g., oxygen tubing and gauze) were stored in open bins in hallways and accessed by $\mathrm{HCP}$ wearing the base PPE layer, and missed opportunities for performing hand hygiene were observed.

A combination of factors that included HCP using multiple gown and glove layers in the COVID-19 unit, extended use of the underlayer of PPE, lapses in cleaning and disinfection of shared medical equipment, and lapses in adherence to hand hygiene likely contributed to widespread $C$. auris transmission. After hospital A removed supplies from hallways, enhanced cleaning and disinfection practices, and ceased base PPE layer practices, no further $C$. auris transmission was detected on subsequent surveys.

The COVID-19 pandemic has prompted facilities to implement PPE conservation strategies during anticipated or existing shortages and to use PPE in ways that are not routine (e.g., extended wear and reuse) (8). Some health care facilities not experiencing shortages allow extra PPE layers because of the perception of increased protection for HCP. CDC does not recommend the use of more than one isolation gown or pair of gloves at a time when providing care to patients with suspected or confirmed SARS-CoV-2 infection $(9,10)$. Such practices among HCP might be motivated by fear of becoming infected with SARS-CoV-2 but instead might increase risks for self-contamination when doffing 
TABLE. Demographic and clinical characteristics of patients colonized with Candida auris in a COVID-19 specialty care unit identified during screening at an acute care hospital $(\mathbf{N}=35)$ - Florida, August 4-18, 2020

\begin{tabular}{lr}
\hline $\begin{array}{l}\text { Characteristic } \\
\text { (no. with available information) }\end{array}$ & No. (\%)* \\
\hline Sex (35) & $14(40)$ \\
Female & $21(60)$ \\
Male & $69(38-101)$ \\
Mean age, yrs (range) (35) & $6(17)$ \\
Clinical culture with C. auris during & \\
admission ${ }^{\dagger}$ (35) & $8(40)$ \\
Mortality within 30 days of screening ${ }^{\S}$ (20) & $2(10)$ \\
Admitted from long-term care facility (20) & \\
Medical devices present at time of screening (20) & $16(80)$ \\
Central venous catheter & $11(55)$ \\
Ventilator & $11(55)$ \\
Nasogastric/Gastric tube & $11(55)$ \\
Urinary catheter & \\
Underlying conditions (20) & $12(60)$ \\
Diabetes & $4(20)$ \\
Chronic wound/wound care & $3(15)$ \\
Malignancy & $3(15)$ \\
Chronic kidney disease & $1(5)$ \\
Chronic lung disease & $1(5)$ \\
Cardiac disease & $4(20)$ \\
No underlying conditions & $5(25)$ \\
Known multidrug-resistant organism & \\
before screening (20) & \\
Vancomycin-resistant Enterococci & $3(15)$ \\
Extended-spectrum beta-lactamase- & \\
producing Enterobacteriaceae & $2(10)$ \\
Methicillin-resistant Staphylococcus aureus & \\
Carbapenem-resistant Enterobacteriaceae & $2(10)$ \\
\hline Abbrevida auris & $0(-)$ \\
$0(-)$ \\
\hline
\end{tabular}

Abbreviation: COVID-19 = coronavirus disease 2019.

* Clinical information available for 20 (57\%) of 35 patients. Medical records for other patients were not available. Clinical information on this subset might not be representative of all patients.

† Results of clinical cultures with Candida auris finalized after colonization was identified by screening during patients' current admission.

$\S$ Contribution of $C$. auris to mortality is unknown.

and for transmission of other pathogens among patients and exacerbate PPE supply shortages. When managing SARS-CoV-2 patients in a dedicated ward, HCP should maintain standard practices (e.g., hand hygiene at indicated times and recommended cleaning and disinfection) intended to prevent transmission of other pathogens. ${ }^{\dagger, \S}$ Outbreaks such as that described in this report highlight the importance of adhering to recommended infection control and PPE practices and continuing surveillance for novel pathogens like C. auris.

\footnotetext{
${ }^{\dagger}$ https://www.cdc.gov/infectioncontrol/guidelines/index.html.

${ }^{\$}$ https://www.cdc.gov/coronavirus/2019-ncov/hcp/infection-control.html.
}

Corresponding author: Christopher Prestel, okn0@cdc.gov.

${ }^{1}$ Division of Healthcare Quality Promotion, National Center for Emerging and Zoonotic Infectious Diseases, CDC; ${ }^{2}$ Bureau of Epidemiology, Division of Disease Control and Health Protection, Florida Department of Health; ${ }^{3}$ Division of Foodborne, Waterborne, and Environmental Diseases, National Center for Emerging and Zoonotic Infectious Diseases, CDC; ${ }^{4}$ Office of the Director, National Institute for Occupational Safety and Health, CDC; ${ }^{5}$ Health Systems and Worker Safety Task Force, CDC COVID-19 Response Team; ${ }^{6}$ Bureau of Public Health Laboratories, Division of Disease Control and Health Protection, Florida Department of Health.

All authors have completed and submitted the International Committee of Medical Journal Editors form for disclosure of potential conflicts of interest. No potential conflicts of interest were disclosed.

\section{References}

1. CDC. Candida auris. Atlanta, GA: US Department of Health and Human Services, CDC; 2020. https://www.cdc.gov/fungal/candidaauris/index.html

2. Tsay S, Welsh RM, Adams EH, et al.; MSD. Notes from the field: ongoing transmission of Candida auris in health care facilities - United States, June 2016-May 2017. MMWR Morb Mortal Wkly Rep 2017;66:514-5. PMID:28520710 https://doi.org/10.15585/mmwr.mm6619a7

3. Vallabhaneni S, Kallen A, Tsay S, et al. Investigation of the first seven reported cases of Candida auris, a globally emerging invasive, multidrugresistant fungus-United States, May 2013-August 2016. Am J Transplant 2017;17:296-9. PMID:28029734 https://doi.org/10.1111/ajt.14121

4. Calvo B, Melo ASA, Perozo-Mena A, et al. First report of Candida auris in America: Clinical and microbiological aspects of 18 episodes of candidemia. J Infect 2016;73:369-74. PMID:27452195 https://doi. org/10.1016/j.jinf.2016.07.008

5. Chowdhary A, Tarai B, Singh A, Sharma A. Multidrug-resistant Candida auris infections in critically ill coronavirus disease patients, India, April-July 2020. Emerg Infect Dis 2020;26:2694-6. PMID:32852265 https://doi.org/10.3201/eid2611.203504

6. Welsh RM, Bentz ML, Shams A, et al. Survival, persistence, and isolation of the emerging multidrug-resistant pathogenic yeast Candida auris on a plastic health care surface. J Clin Microbiol 2017;55:2996-3005. PMID:28747370 https://doi.org/10.1128/JCM.00921-17

7. Adams E, Quinn M, Tsay S, et al.; Candida auris Investigation Workgroup. Candida auris in healthcare facilities, New York, USA, 2013-2017. Emerg Infect Dis 2018;24:1816-24. PMID:30226155 https://doi.org/10.3201/eid2410.180649

8. CDC. Coronavirus disease 2019 (COVID-19): optimizing supply of PPE and other equipment during shortages. Atlanta, GA: US Department of Health and Human Services, CDC; 2020. https://www. cdc.gov/coronavirus/2019-ncov/hcp/ppe-strategy/general-optimizationstrategies.html

9. CDC. Coronavirus disease 2019 (COVID-19): strategies for optimizing the supply of isolation gowns. Atlanta, GA: US Department of Health and Human Services, CDC; 2020. https://www.cdc.gov/ coronavirus/2019-ncov/hcp/ppe-strategy/isolation-gowns.html

10. CDC. Coronavirus disease 2019 (COVID-19): strategies for optimizing the supply of disposable medical gloves. Atlanta, GA: US Department of Health and Human Services, CDC; 2020. https://www.cdc.gov/ coronavirus/2019-ncov/hcp/ppe-strategy/gloves.html 